\title{
Could a more-work-strategy (MWS) do better than a lockdown strategy in developing countries during the COVID-19 pandemic?
}

\author{
Muhammad Irfanullah Siddiqui, ${ }^{*}$ Adeel Ahmed Khan, ${ }^{\dagger}$ and Fahad Saqib Lodhi ${ }^{\ddagger}$
}

\section{Dear Editor,}

The COVID-19 pandemic has the potential to create devastating social, economic, and political crises. Globally, multiple public health strategies have been implemented to control the spread of this virus. The purpose of these strategies is to prevent infection and delay exposure in order to give health systems enough time to prepare themselves for the rising number of patients.

Current strategies typically focus on hand washing, wearing of masks, and physical distancing (Güner et al., 2020). To achieve physical distancing, most countries adopted a policy of partial or complete lockdown. In such lockdowns, much of the focus has been on closing places like schools, places of worship, shopping malls, and workplaces and reducing mixing in the general community (Prem et al., 2020). For most countries, this has resulted in economic collapse, with a threat of many more deaths resulting from hunger and chaos-for some, potentially a greater number than from the virus itself (Buheji et al., 2020). This halt of economic and social activities has also led to ill effects on the mental health of the population, which may lead to anxiety, depression, crime, and suicide (Zhang \& Ma, 2020). Some countries, like Sweden, South Korea, and Taiwan, did not opt for lockdowns but focused on hand washing, use of masks, and voluntary physical distancing (An \& Tang, 2020).

In partial lockdown, people try to do all their work in the typically allocated workday schedule, which is usually 8 to 10 hours a day. There is often a sense of panic among people that shopping and other commodities will be closed after their dedicated work time, and they try to finish 24-hour assignments in just 8 to 10 hours. Consequently, the entire population may go out into the community at the same time, during limited hours, and there is an undesirable rush of people at the same place resulting in severe overcrowding. This phenomenon can be illustrated using the following example:

In normal times, a moderate-sized shopping mall is typically open for 12 hours a day, during which time it serves 3,600 people. Hence, the mall handles 300 customers per hour (i.e., 3,600/12) and a total of 25,200 customers per week (3,600 $\times 7$ ). During curfew conditions (lockdown), the same mall will open, say, for six hours a day. After one week, most of the people using the mall need to buy food and essential items and will be forced to attend the mall when they are finished with other time-restricted activities. Hence, we could see all 25,200 people trying to approach the mall to meet their needs in a compressed timeframe, as they are never sure what the situation may be the next day, whether the mall will be open or not, and whether they will be able to meet their needs. Hence, there would be severe overcrowding. The mall will now have to handle 4,300 persons per hour (i.e., 25,200/6), as compared with 300 per hour in routine times, resulting in overcrowding and an increased threat of very fast spread of the disease (Siddiqui, 2020).

Neither the China model of a complete lockdown, nor the Sweden model of voluntary physical distancing are readily applicable in densely populated developing countries, due to additional considerations arising from poor literacy rates and overcrowding as a routine phenomenon in these countries.

In such environments, common to many low- and middle-income countries (LMIC's), we are proposing changing from the lockdown strategy to a more-work-strategy (MWS) to achieve physical distancing at these overcrowded places. All places of public interest should be open 24 hours a day (168 hours a week). This will help alleviate panic in the community, and residents and business would be more confident in their ability to get their work and routine shopping duties done without fear of closures. Workforce management in offices and supermarkets would be required in order to manage the working hours of 24 hours a day. One of the options would be to divide the workforce into three shifts, which would also help in maintaining physical distancing, and the workload would also be distributed across these time frames. Consider the following example:

Suppose in a bank there are 24 employees working eight hours per day. Divide them into three shifts of eight persons per shift of eight hours. This reduction in staff during any one shift will help increase the distance between staff members by

Correspondence to: Adeel Ahmed Khan, Faculty, Saudi Board Program of Preventive Medicine, Ministry of Health, Street Abdul Aziz Al-Rafai, Al - Awali, Mecca, 24371, Saudi Arabia. E-mail: adeel_ahmed_khan@hotmail.com

To cite: Siddiqui, M. I., Khan, A. A., \& Lodhi, F. S. (2020). Could a more-work-strategy (MWS) do better than a lockdown strategy in developing countries during the COVID-19 pandemic? Journal of Community Safety and Well-Being, 5(3), 133-134. https://doi.org/10.35502/jcswb.155

@ Author(s) 2020. Open Access. This work is distributed under the Creative Commons BY-NC-ND license. For commercial re-use, please contact sales@sgpublishing.ca.

gPUBLISHING Published by SG Publishing Inc. CSKA Official publication of the Community Safety Knowledge Alliance. 
a factor of three compared with a normal working schedule. Moreover, the clients will also be distributed across three shifts, reducing the load of clients to one-third of the normal working day pattern. This could be further reduced by giving online appointments to two-thirds of clients so that they would actually attend the bank only at times designated for them. One-third of appointments could be reserved for emergency cases, which could be served on a first-come-firstserved basis.

These steps could help reduce overcrowding in many public environments, enabling such communities to more easily maintain recommended physical distancing. For these changes in timing, revised standard operating procedures (SOPs) would also need to be developed, since working hours might also include night hours. Deployment of law enforcement agents at night-time workplaces might also be considered. For big cities and very busy offices, a three-shift option would work, while for smaller cities, a two-shift option may be sufficient.

Hospital administrators in many developing countries are already dealing with COVID-19 cases by demanding 24hour rotations by public service healthcare workers (HCWs), due to high workload in facilities. These frontline professionals are at risk of experiencing burnout and are highly vulnerable to physical exhaustion, fear, sleep problems, and emotional disturbances (Spoorthy et al., 2020). Also, due to these excessive working hours, their immune system may suffer, increasing their risk for COVID-19, which will potentially take them out of the health system, where they are badly needed. As a result, the vulnerable healthcare system in developing countries could face severe HCW shortages and a reduced number of healthcare facilities during this critical phase of the pandemic.

We propose to involve the private HCWs, as $90 \%$ of them are working in the private sector in developing countries. This important workforce is usually at home and not working actively due to the current crisis. Governments may be able to offer them work in high-burden COVID-19 hospitals, with good remuneration including a high salary, promises of awards and certificates, and also permanent positions in the public sector once this crisis is over. This will help reduce the duty hours of public sector HCWs, who, as a result, would be have a greater likelihood of enjoying adequate rest, helping them to retain their immunity status. Healthcare systems would gain a revival from this new workforce, and it would be motivating for those HCWs who are currently becoming exhausted due to the relentless workload.

In summary, the complete lockdown strategy for COVID-19 control in developing countries may not be possible for a prolonged period of time due to their troubled economies and other social factors. Also, partial lockdowns may easily backfire as physical distancing cannot be maintained during reduced functioning hours. We propose MWS for South Asian and sub-Saharan African countries, which can apply the concept of MWS by rotating their workforce over various shifts. This will eventually result in boosting the economy of these countries, as it will help employers to have continuing and even substantial growth. Regarding healthcare systems, private HCWs should be appointed, as a force multiplier for the public sector, to meet growing strains and staff deficiencies and to be able to prepare for more adverse scenarios yet to come.

\section{CONFLICT OF INTEREST DISCLOSURES}

The authors have no conflicts of interest to declare.

\section{AUTHOR AFFILIATIONS}

*Professor, Department of Community Medicine and Public Health, Faculty of Medicine, Umm Al-Qura University, Makkah, Saudi Arabia; ${ }^{\dagger}$ Faculty, Saudi Board Preventive Medicine, Ministry of Health, Mecca, Saudi Arabia; 任rofessor of Epidemiology, Department of Community Medicine, Women Medical and Dental College Abbottabad, Pakistan.

\section{REFERENCES}

An, B. Y., \& Tang, S. Y. (2020). Lessons from COVID-19 responses in East Asia: Institutional infrastructure and enduring policy instruments. The American Review of Public Administration, 0275074020943707. doi: 10.1177/0275074020943707.

Buheji, M., da Costa Cunha, K., Beka, G., Mavric, B., de Souza, Y. L., da Costa Silva, S. S., ... \& Yein, T. C. (2020). The extent of the COVID-19 pandemic socio-economic impact on global poverty. A global integrative multidisciplinary review. American Journal of Economics, 10(4), 213-224.

Güner, H. R., Hasanoglu, I., \& Aktas, F. (2020). COVID-19: Prevention and control measures in the community. Turkish Journal of Medical Sciences, 50(SI-1), 571-577. doi: 10.3906/sag-2004-146.

Prem, K., Liu, Y., Russell, T. W., Kucharski, A. J., Eggo, R. M., Davies, N., \& Abbott, S. (2020). The effect of control strategies to reduce social mixing on outcomes of the COVID-19 epidemic in Wuhan, China: A modelling study. The Lancet Public Health. doi: 10.1016/ S2468-2667(20)30073-6

Siddiqui, M. I. (2020). New strategy for management of COVID-19 MWS Part $C$ consequences of lockdown [YouTube video]. Retrieved from: https://www.youtube.com/watch? $v=t w-89 Q 9 D s 64$.

Spoorthy, M. S., Pratapa, S. K., \& Mahant, S. (2020). Mental health problems faced by healthcare workers due to the COVID-19 pandemic-A review. Asian Journal of Psychiatry, 51, 102119. doi: 10.1016/i.ajp.2020.102119.

Zhang, Y., \& Ma, Z. F. (2020). Impact of the COVID-19 pandemic on mental health and quality of life among local residents in Liaoning Province, China: A cross-sectional study. International Journal of Environmental Research and Public Health, 17(7), 2381. doi: 10.3390/ ijerph17072381. 